\title{
Derailed Cardiac Surgical Practice during COVID-19 Pandemic: Concerns and Proposed Solution Strategy
}

\author{
Prateek Vaswani ${ }^{1}$ Manoj Kumar Sahu² \\ ${ }^{1}$ Department of Cardiothoracic Surgery, Cardiothoracic and \\ Neurosciences Centre, All India Institute of Medical Sciences, \\ New Delhi, India \\ Intensive Care for CTVS, CT Centre, All India Institute of Medical \\ Sciences, New Delhi, India
}

\begin{abstract}
Address for correspondence Manoj Kumar Sahu, MD, DNB, Intensive Care for CTVS, Cardiothoracic and Neurosciences Centre, Cardiothoracic and Neurosciences Centre, All India Institute of Medical Sciences, CTVS Office, Room No 5, $7^{\text {th }}$ Floor, Ansari Nagar, New Delhi, 110029, India (e-mail: drmanojsahu@gmail.com).
\end{abstract}
Abstract
Keywords
- COVID 19
- cardiac surgery
- ethics
- RT-PCR test
- preparedness

The current ongoing novel corona virus disease 2019 (COVID 19) pandemic has led to reallocation of substantial hospital resources and workforce depriving the institution's ability to handle the routine caseload. The sustenance of health care would require a comprehensive management plan and focused strategy. The cardiac surgical department would consume majority of critical care resources, hence, a balance should be sought between the resumption of routine surgeries and the emergency unavoidable ones. We have proposed an alternative plan to guide the formulation of a strategic comeback.

\section{Introduction}

Since the arrival of coronavirus disease 2019 (COVID 19) and its declaration as pandemic, India has undergone five phases of lockdown, the present fifth being unlock one. The highly contagious disease is spreading relentlessly throughout the country despite all these containment measures. The end of a nationwide total lockdown with beginning of unlocking of the activities at both sociocultural and economic fronts has started in a graded manner. There is a growing demand for stratification, organization, and expeditious preparedness at health care centers in these times of crisis for a smooth resumption of services.

\section{What to Expect?}

The need for multidisciplinary efforts with decision taking the local and national pandemic load into account cannot be emphasized further. There should be an adequate flexibility in readjusting the protocols to respond to the evolving COVID 19 turmoil with ongoing change in diagnostic and therapeutic strategies. The awareness of an imminent surge must be kept in mind. This may be further complicated with super-added or coexisting pathologies and/or exacerbation of a chronic pulmonary illness. We believe that a set of protocols if formulated could provide for effective tide over of this unavoidable and unforeseen calamity.

The prime concern begins with control of pandemic within the community. The availability of facility for physical isolation in the hospital, declining incidences in COVID 19 in the vicinity, and creation of adequate resources for performance of nonemergent cases is a prerequisite. A backup management plan should be jotted down in case of resurgence of cases.

The guidelines from Lombardy region in Northern Italy have divided health care institutions into "Hub Centers" for active cardiac interventions and "Spoke centers" as satellites working for scrutinizing the enrolment of patients who published online

February 24, 2021
DOI https://doi.org/

10.1055/s-0041-1723623

ISSN 2457-0206. (c) 2021. Official Publication of The Simulation Society (TSS), accredited by International Society of Cardiovascular Ultrasound (ISCU).

This is an open access article published by Thieme under the terms of the Creative Commons Attribution-NonDerivative-NonCommercial-License, permitting copying and reproduction so long as the original work is given appropriate credit. Contents may not be used for commercial purposes, or adapted, remixed, transformed or built upon. (https://creativecommons.org/licenses/by-nc-nd/4.0/) Thieme Medical and Scientific Publishers Pvt. Ltd., A-12, 2nd Floor, Sector 2, Noida-201301 UP, India 
cannot be delayed further for intervention, taking care of chronically debilitated patients, surgical site wound infections or dehiscence, keeping the nonemergent cases aware of hospital policies, special support with policy for utmost level of care for the working personnel, and keeping records. ${ }^{1}$ These Spoke centers have special units for keeping records of radiological investigations, serum marker levels, pathological findings, and COVID 19-associated complications developing early or late in the course of the disease. The importance of record keeping cannot be less emphasized in disaster preparedness.

1. There has been observed cardiovascular associations in up to $30 \%$ cases with $11.8 \%$ having significant myocardial injury with elevated troponin levels., ${ }^{2,3}$

2. Acute respiratory distress syndrome is a frequent sequela of COVID 19 with an observed incidence of $20 \%$, median 8 days from onset of symptom with requirement of mechanical ventilation in $12.3 \%$ of them as revealed in study of 138 patients by Wang et $\mathrm{al}^{4}$ with other studies reporting ventilator requirements between 12 and $24 \%$ cases. $^{5,6}$

3. Occurrence of arrhythmias, acute myocardial insult, and cardiogenic shock has been observed in 17, 7, and 9\% cases, respectively, by Wang et $\mathrm{al}^{4}$ which has been correlated well by other studies.<sup $>^{7,8}$ In severe cases, one-third have been observed developing cardiomyopathy. ${ }^{9}$

4. Thromboembolic complications such as deep vein thrombosis, pulmonary embolism, and stroke have been revealed even in younger subset ( $<50$ years) in the absence of risk associations. The resultant hypercoagulability that develops in the due course of disease has been substantiated in postmortem studies. Menter et al in their postmortem of 21 patients found significant pulmonary embolus in 4 with microthrombi in alveolar capillaries in $45 \%$ cases with revealed histology and 3 patients having microangiopathy of glomerular capillaries. ${ }^{10}$ The incidence of deep vein thrombosis has been found in $58 \%$ cases with involvement of both sides by Wichmann et al preceded by the serological elevation of D-dimers. ${ }^{11}$ The pulmonary pathological study by Ackermann et al demonstrated endotheliitis among other findings. ${ }^{12}$ This severe endothelial injury can propagate hypercoagulopathy in accordance with Virchow's triad. The incidence of stroke varies from 1.91\% in patients not requiring critical care ${ }^{13}$ to 2 to $3.7 \%$ cases admitted in the intensive care unit (ICU) even with anticoagulation. ${ }^{14,15}$ Oxley et al found stroke in 5 patients, all less than 50 years of age over a 14-day observation interval when the rates in prepandemic era was to the tune of 0.7 large vessel strokes over 2-week period in those under 50 years of age. ${ }^{16} \mathrm{~A}$ study by Bellosta et al reported $16 \%$ incidence of acute limb ischemia with $90 \%$ males in the 7th decade with $71 \%$ successful revascularization which was lower than institution's expectations, and although these patients did not require reintervention in the postoperative period while maintained on heparin, role of heparin could not be established. ${ }^{17}$ Bleeding complications of COVID 19 have been seldom reported. ${ }^{14}$
5. The occurrence of a storming cytokine response with elevations in proinflammatory cytokines (interleukin-6), serum levels of D-dimers with ferritin, and clinical pyrexia has been demonstrated with increasing severe disease course culminating in mortality. ${ }^{2,18}$

\section{The Scary Hospital Environment}

There is a striking fear among patients while entering the hospital. The hospital environment should be conducive with minimal risk of COVID 19 exposure. Attention should be paid on availability of supplies, food sanitation, drug availability, personal protective equipment (PPE), management algorithms, and interaction among patients and visitors with health care workers. The formulation of plan is essential for the same which emphasizes upon limited movement and exposure to the ones admitted. There should be reassessment of policy for repeated laboratory workups, avoiding unnecessary imaging, need for examining these patients, and shifting the patients inside the hospital premises. Family and relatives should be contacted with teleconferencing. The informed consent should also familiarize the relatives and guardians with these protocols.

\section{Institutional Care Provision}

The creation of separate management strategies to cater for COVID 19 and requirements of non-COVID should be made. Escalating routine procedures will encompass the entire available facilities, so protocols for judicious utilization should be stressed upon.

As far as feasible when admission is deemed necessary, these individuals should be admitted in proven negative areas with minimal transmission risk. The counseling regarding limitation of visitors and the possibility of surgery being cancelled should be explained whenever appropriate preferably before admission. Social distance norms (2 feet) should be strictly adhered to in various zones of the hospital. Hand sanitization facility and temperature screening should be made available at the point of care of importance like entry into hospital and/or other working areas. Donning of the N95 mask or equivalent must be firmly implemented in the hospital premises. Assessment should be made at the time of admission for anticipated need of follow-up care in case services are suspended in face of resurgence. Policy for early discharge should be made after an uneventful postoperative course and speedy recovery. Retesting with reverse transcriptase polymerase chain reaction (RT-PCR) before patient discharge from the ICU/hospital depending on hospital policy should be considered. Education of the relatives, parents, or guardians (in case of pediatric patients) regarding home care of their patients must be actively considered especially so in patients with prolonged hospital stay.

The institutional development and adoption of plan for screening patients with resumption of elective surgeries is essential. Following can be considered in this regard:

- Teleconferencing with institution-approved set of questions for early identification. 
- The provision of a separate room for admission and testing for COVID 19 with facility to keep the patient there till a negative report is obtained.

- The negative testing with transfer to ward does not end the process and screening should be carried to ward with daily assessment and COVID 19 test whenever deemed necessary.

\section{Workforce is Priority}

Hospital personnel awareness regarding keeping themselves safe should be prioritized. The strict maintenance of proper hand and personal hygiene, disinfecting working areas, judicious use of PPE, creating safe areas, physical distance adherence, and isolating oneself in case of contracting COVID 19 is essential for minimizing hospital-acquired transmission. The success of restarting of program is borne by health personnel's adherence to these protocols laid by institutions in case of COVID 19 exposure. A comprehensive plan with mitigation of staff should be available.

The need for a conducive environment, motivational support, and boost cannot be emphasized further for ensuring mental health of health care personnel. Incessant endeavors in establishing meetings among members of the surgical team, intensivists, administrative personnel, and public health officers is need of the hour for efficient handling of increasing case load. This would facilitate exchange of ideas for contemplating a comprehensive plan of management.

\section{The Ethical Jostle and Conundrum}

In 2014, the American College of Chest Physicians published a consensus statement for caring the critically ill patients during times of overwhelming pandemic force. ${ }^{19}$ The jeopardy during this unexpected situation should not be a justification for failure to comply with the ethical guide. There are certain proposed solutions to this problem:

- It should be understood that laid down ethical criteria do not differentiate between the withdrawal and withholding of the essential services in these difficult times.

- Allocation of limited facilities should be triage-based.

- Ethics must allow for exclusion criteria to be employed wherever necessary based on clinician's judgment.

- Reallocation of facilities to those in dire need after withdrawing from the stable patients should be permitted by ethical standards.

- In crisis times, ethics should not allow to distinguish the allocation of these services between health care personnel and the general public.

- The hectic schedule must have relaxations and provisions for assistance of health care workers as they are the mainstay workforce fighting upfront in this overwhelming situation.

- The clinicians who do not abide by this crisis policy should assume the nonclinical or support group positions, however, should mandatorily participate with no exclusion whatsoever.
- Involvement and inputs must be taken from clinicians and health care personnel. They must also be encouraged to implement measures for protection of self and their close ones.

- The provision for "Do Not Actively Resuscitate" should be incorporated based on clinical judgment.

\section{Judicious COVID 19 Testing}

RT-PCR testing must be done in cases from hotspots, high-risk exposure to laboratory-confirmed cases, or presence of symptoms; however, if feasible all patients being admitted or considered for surgery should undergo testing. We can expect evolving newer serology testing strategies (antibody titer testing for immunoglobulin $\mathrm{G}$ and immunoglobulin $\mathrm{A}$ ) for screening healthy and asymptomatic personnel as the pandemic evolves further. This will smoothen the process of reporting and in decision making for triage. Meanwhile, it should be realized that the false negativity of current available tests prevent a confirmed exclusion. This fact must always be kept in mind while dealing with these patients with a few of the following:

- All cases being admitted should undergo RT-PCR preferably with minimal interval of time from planned procedure (24-48 hours before planned surgery).

- In case of laboratory-confirmed positive asymptomatic cases, surgery should be delayed/postponed up to 2 weeks.

- Cautious interpretation should be done in patients without symptoms as 30 to $50 \%$ are falsely negative. ${ }^{20,21}$ This rate may escalate with improper sample collecting technique or during early phase of the disease when viral load is insufficient. ${ }^{22}$ In case of high index of suspicion or transfer from a hotspot area, assessment by infectious disease personnel and testing again after 24 to 48 hours can be considered.

- Even after initial testing if there is doubt on clinical examination, isolation for 10 to 14 days should be considered with option for retesting whenever required.

- If development of symptoms and signs are observed in postoperative period with concerns for COVID 19, repeat RT-PCR (nasal, pharyngeal, or tracheal specimen) and/or radiological imaging (preferably computed tomography chest) should be considered. ${ }^{23}$

- Till the time a conclusive exclusion test with high negative predictive value is unavailable, the above norms should be strictly adhered for ensuring safety of all.

\section{Departmental Preparedness Policy}

The resumption of routine functions must be phasic depending upon institution's adoptability and considering major proportions of hospital beds and critical care beds with support gadgets like ventilators and monitors are being dedicated as COVID 19 areas with limited overall resources. The cardiac surgery units must be in alliance with the hospital phasic transition.

To ensure adequate control, intradepartmental formulation of teams who focus on preventing transmission, 
identifying postsurgery sequelae of COVID 19 or other similar illnesses, assessing workforce inadequacy, and quality assessment points should be constituted.

Multidisciplinary discussions among cardiologist, cardiac surgeons, and intensive care specialists should be held constructively debating on comprehensive cardiac diagnosis, risk of delaying the surgery, and discussion regarding precautions against hospital-acquired COVID 19 or other infectious diseases. The list of cases must be decided prehand with an order, prioritizing cases which will be given preference or will be delayed in case of an overwhelming situation that occupies the entire hospital capacity and engages majority of hospital personnel.

The waiting list people may deteriorate with these delays. Reassessment should be made frequently with stratification of these patients. There has been a shift in management strategy to medical approach or less invasive ones like percutaneous interventions-based regimens from open cardiac surgeries in the face of the pandemic. The following may be applicable in this regard:

- Video calls, text messages, phone calls, etc. at decided time intervals for assessment with proper instructions in case of worsening symptoms should be done.

- Availability of a well laid out protocol for high-risk cases or in those with expected clinical worsening is essential.

- Multidisciplinary meeting with discussions regarding high-risk or complex anatomical diagnosis should be held.

- Frequent reassessment of local disease load trends with corresponding alterations in hospital devised policy should be undertaken.

\section{Conclusion}

The utmost level of care by cardiac surgical unit in an unexpected unforeseen COVID 19 pandemic requires comprehensive and multidisciplinary efforts. The growing number of waiting patients and those with cancellations, experience both physical and mental stress which is a matter of great concern. The ethical conundrum cannot be completely disregarded; however, place for a few adjustments can be considered in such times. The institution's approach in management varies but should be uniformly standardized with dynamic change in policies with provision for inputs by medical personnel and a backup plan formulation in case of resurgence. The phrase united we stand and divided we fall is of relevance here.

The financial impact on various health care centers cannot be entirely substantiated. The pandemic updates in both local and national fronts must be catered to before formulation of the institutional algorithm. This herculean task will require cardiac surgeons to evolve with a strict focus on the principles laid out in the STS Adult Cardiac Triage preamble which revolve around the protection of patients as the foremost priority, health workers themselves with their fellow colleagues, and last but not the least protecting the overall society. ${ }^{24}$ The realization that COVID 19 patients will continue to be a part of the hospital in times to come till a vaccine or herd immunity is developed is necessary for everyone involved in the health care process. There is no running from this fact.

\section{Source of Financial Support}

None.

\section{Conflict of Interest}

None declared.

\section{References}

1 Bonalumi G, di Mauro M, Garatti A, Barili F, Gerosa G, Parolari A; Italian Society for Cardiac Surgery Task Force on COVID-19 Pandemic. The COVID-19 outbreak and its impact on hospitals in Italy: the model of cardiac surgery. Eur J Cardiothorac Surg 2020;57(6):1025-1028

2 Huang C, Wang Y, Li X, et al. Clinical features of patients infected with 2019 novel coronavirus in Wuhan, China. Lancet 2020;395(10223):497-506

3 Zheng YY, Ma YT, Zhang JY, Xie X. COVID-19 and the cardiovascular system. Nat Rev Cardiol 2020;17(5):259-260

4 Wang $\mathrm{D}, \mathrm{Hu} \mathrm{B}, \mathrm{Hu} \mathrm{C}$, et al. Clinical characteristics of 138 hospitalized patients with 2019 novel coronavirus-infected pneumonia in Wuhan, China. JAMA 2020;323(11):1061-1069

5 Richardson S, Hirsch JS, Narasimhan M, et al; the Northwell COVID-19 Research Consortium. Presenting characteristics, comorbidities, and outcomes among 5700 patients hospitalized with COVID-19 in the New York City Area. JAMA 2020;323(20):2052-2059

6 Petrilli CM, Jones SA, Yang J, et al. Factors associated with hospital admission and critical illness among 5279 people with coronavirus disease 2019 in New York City: prospective cohort study. BMJ 2020;369:m1966

7 Cao J, Tu WJ, Cheng W, et al. Clinical features and short-term outcomes of 102 patients with coronavirus disease 2019 in Wuhan, China. Clin Infect Dis 2020;71(15):748-755

8 Chen $\mathrm{T}, \mathrm{Wu} \mathrm{D}$, Chen $\mathrm{H}$, et al. Clinical characteristics of 113 deceased patients with coronavirus disease 2019: retrospective study. BMJ 2020;368:m1091

9 Arentz M, Yim E, Klaff L, et al. Characteristics and outcomes of 21 critically ill patients with COVID-19 in Washington State. JAMA 2020;323(16):1612-1614

10 Menter T, Haslbauer JD, Nienhold R, et al. Postmortem examination of COVID-19 patients reveals diffuse alveolar damage with severe capillary congestion and variegated findings in lungs and other organs suggesting vascular dysfunction. Histopathology 2020;77(2):198-209

11 Wichmann D, Sperhake J-P, Lütgehetmann M, et al. Autopsy findings and venous thromboembolism in patients with COVID-19: a prospective cohort study. Ann Intern Med 2020; 173(4):268-277

12 Ackermann M, Verleden SE, Kuehnel M, et al. Pulmonary vascular endothelialitis, thrombosis, and angiogenesis in Covid-19. N Engl J Med 2020;383(2):120-128

13 Lodigiani C, Iapichino G, Carenzo L, et al; Humanitas COVID-19 Task Force. Venous and arterial thromboembolic complications in COVID-19 patients admitted to an academic hospital in Milan, Italy. Thromb Res 2020;191:9-14

14 Helms J, Tacquard C, Severac F, et al; CRICS TRIGGERSEP Group (Clinical Research in Intensive Care and Sepsis Trial Group for Global Evaluation and Research in Sepsis). High risk of thrombosis in patients with severe SARS-CoV-2 infection: a multicenter prospective cohort study. Intensive Care Med 2020;46(6):1089-1098

15 Klok FA, Kruip MJHA, van der Meer NJM, et al. Incidence of thrombotic complications in critically ill ICU patients with COVID-19. Thromb Res 2020;191:145-147 
16 Oxley TJ, Mocco J, Majidi S, et al. Large-vessel stroke as a presenting feature of Covid-19 in the young. N Engl J Med 2020; 382(20):e60

17 Bellosta R, Luzzani L, Natalini G, et al. Acute limb ischemia in patients with COVID-19 pneumonia. J Vasc Surg 2020; S0741-5214(20):31080-31086

18 Mehta P, McAuley DF, Brown M, Sanchez E, Tattersall RS, Manson JJ; HLH Across Speciality Collaboration, UK. COVID-19: consider cytokine storm syndromes and immunosuppression. Lancet 2020;395(10229):1033-1034

19 Biddison LD, Berkowitz KA, Courtney B, et al; Task Force for Mass Critical CareTask Force for Mass Critical Care. Ethical considerations: care of the critically ill and injured during pandemics and disasters: CHEST consensus statement. Chest 2014;146(4, Suppl):e145S-e155S

20 Kucirka LM, Lauer SA, Laeyendecker O, Boon D, Lessler J. Variation in false-negative rate of reverse transcriptase polymerase chain reaction-based SARS-CoV-2 tests by time since exposure. Ann Intern Med 2020;173(4):262-267

21 West CP, Montori VM, Sampathkumar P. COVID-19 testing: the threat of false-negative results. Mayo Clin Proc 2020; 95(6):1127-1129

22 Cheng MP, Papenburg J, Desjardins M, et al. Diagnostic testing for severe acute respiratory syndrome-related coronavirus 2 : a narrative review. Ann Intern Med 2020;172(11):726-734

23 Long C, Xu H, Shen Q, et al. Diagnosis of the coronavirus disease (COVID-19): rRT-PCR or CT? Eur J Radiol 2020;126:108961

24 Haft JW, Atluri P, Ailawadi G, et al; Society of Thoracic Surgeons COVID-19 Task Force and the Workforce for Adult Cardiac and Vascular Surgery. Adult cardiac surgery during the COVID-19 pandemic: a tiered patient triage guidance statement. Ann Thorac Surg 2020;110(2):697-700 